\title{
Corela
}

Cognition, représentation, langage

14-1 | 2016

Vol. $14, n^{\circ} 1$

\section{La notion de lieu de corpus : un nouvel outil pour l'étude des terrains numériques en linguistique}

\section{Laetitia Emerit}

\section{OpenEdition}

Journals

Édition électronique

URL : http://journals.openedition.org/corela/4594

DOI : $10.4000 /$ corela.4594

ISSN : 1638-573X

Éditeur

Cercle linguistique du Centre et de I'Ouest - CerLICO

Référence électronique

Laetitia Emerit, « La notion de lieu de corpus : un nouvel outil pour l'étude des terrains numériques en linguistique », Corela [En ligne], 14-1 | 2016, mis en ligne le 16 juin 2016, consulté le 01 mai 2019. URL : http://journals.openedition.org/corela/4594 ; DOI : 10.4000/corela.4594

Ce document a été généré automatiquement le 1 mai 2019.

\section{(c) (1) (3) (-)}

Corela - cognition, représentation, langage est mis à disposition selon les termes de la licence Creative Commons Attribution - Pas d'Utilisation Commerciale - Partage dans les Mêmes Conditions 4.0 International. 


\title{
La notion de lieu de corpus : un nouvel outil pour l'étude des terrains numériques en linguistique
}

\author{
Laetitia Emerit
}

1 Dans cet article nous proposerons la notion de « lieu de corpus » comme une alternative complémentaire à celle de « corpus ». Le « lieu de corpus » doit s'envisager comme un lieu à partir duquel il est possible de créer des corpus numériques et jusqu'auquel il est nécessaire de remonter pour interpréter ces corpus.

2 Pour définir et illustrer la notion de lieu de corpus nous nous appuierons sur la description du compte Facebook «Ma Thèse Sdl ». Ce lieu de corpus a été créé dans le cadre de notre thèse de doctorat dont l'objectif était d'observer le discours produit sur le réseau social numérique Facebook à l'occasion notamment des souhaits d'anniversaires.

Pour atteindre ce but il a été nécessaire de bâtir une méthodologie permettant d'en rendre compte et qui commence avec la notion de lieu de corpus. Notion que dont nous proposerons une définition ici et qui demande de considérer l'instabilité du discours apparaissant dans les environnements numériques comme constitutive de cet objet de recherche et donc de ses potentiels " échantillons".

4 La notion de lieu de corpus n'exclut pas celle de corpus, celui-ci devient une potentialité qui lui est subordonnée. À partir d'un lieu de corpus il sera possible de créer plusieurs corpus différents qui seront constitués de figements focalisés sur une partie des données accessibles. Nous proposerons dans cet article de représenter cette hiérarchisation au travers d'une modélisation arborescente.

5 Cette position demande de remettre en cause la position du chercheur et une partie de son outillage théorique et méthodologique. Elle nous interroge également sur la place des locuteurs-scripteurs. Comment les relations et le positionnement du chercheur et des locuteurs scripteurs influencent-ils la construction du corpus? Et quelle position déontologique adopter pour recueillir les données d'un corpus semi-privé ? 
6 Pour répondre à ces questions nous commencerons par définir le lieu de corpus, ce qui nous amènera à présenter ses caractéristiques et à définir la notion de corpus idionumérique qui lui est attachée. Ensuite nous traiterons la question de la représentation du lieu de corpus en proposant le modèle de la représentation arborescente tel que nous l'avons utilisé dans le cas du lieu de corpus « Ma Thèse Sdl ». Enfin nous proposerons une réflexion sur la place et le rôle des différents acteurs du lieu de corpus (environnement-locuteurs-chercheur) et leurs possibles interactions.

\section{La question du corpus en linguistique}

7 La question du corpus en linguistique étant régulièrement posée, dans cet article nous tenterons de la dépasser afin de l'adapter aux particularités des terrains numériques. Cette ouverture est nécessaire car les données nativement numériques ne peuvent conserver leurs propriétés technolangagières (Paveau 2012) qu'en les considérant comme partie prenante du corpus.

8 En linguistique, le terme "corpus » est polysémique et renvoie, comme le soulignent Gadet et Copeau (2007: 101), à différents degrés d'ouverture :

«Il y a des définitions larges (comme chez Dalbera (2002) : ensemble d'éléments sur lequel se fonde l'étude d'un phénomène linguistique), qui qualifient tous les matériaux qu'utilise le linguiste, en particulier ce que l'on peut appeler son corpus de recherche (les séquences jugées pertinentes pour la description d'un phénomène). D'autres définitions plus ciblées (collection ordonnée d'enregistrements de productions linguistiques orales et multimodales, chez Baude 2006) conviennent mieux aux actuels grands corpus informatisés. »

9 L'opposition entre définitions "larges » et "ciblées " proposée par Gadet et Copeau (2007) permet d'appréhender la notion de corpus en linguistique comme un continuum de forme et de taille au sein duquel chaque linguiste doit se situer en fonction de son terrain.

La définition de Sinclair (1996:4) ajoute une dimension pré-analytique :

«Un corpus est une collection de données langagières qui sont sélectionnées et organisées selon des critères linguistiques explicites pour servir d'échantillon du langage. »

11 On peut considérer que la définition de Sinclair est « ciblée » selon les critères de Gadet et Copeau. Elle prend également en compte l'intervention du linguiste dans la construction du corpus. Pourtant si on applique cette définition dans le cadre du discours numérique il faut travailler à partir de données langagières stabilisées, c'est-à-dire d'extractions ou, au mieux, de captures d'écran. Dans ce cas le terrain premier, c'est-à-dire l'environnement numérique (le « contexte ») où se situe la recherche, est exclu de la notion de corpus.

Pourtant, comme le soulignent Rastier et Pincemin (1999: 85) :

« Le contexte n'est pas une réalité à côté du texte, dont la prise en compte serait un raffinement, intervenant sur le mode d'ajouts ou de corrections. Le contexte est l'extériorité qui dessine le texte. »

13 Bénédicte Pincemin, dans sa thèse, va plus loin en affirmant que :

« Le corpus fournit à la fois des éléments à étudier, mais aussi l'environnement descriptif de ces éléments. Le corpus est un tout, un vaste ensemble, qui constitue à lui seul le cadre et le référentiel de l'analyse. » (Bommier-Pincemin 1999 : 415)

Le contexte et le texte peuvent alors être considérés comme appartenant, comme définissant, le corpus en linguistique. Il nous semble pourtant que cette prise en compte 
du contexte, tout en lui donnant une place à l'intérieur du corpus, le rejette tout de même comme un phénomène périphérique au texte.

Ici, si le contexte est définitoire du texte il lui reste extérieur, ors, ce que nous observons dans les environnements numériques c'est une réelle interconnexion entre le contexte et le texte qu'il nous semble plus adéquat d'aborder sous l'angle environnemental:

«Une langue est ancrée de manière multiple dans une société ou une culture, et ces ancrages se manifestent d'une manière ou d'une autre dans les discours, dans les interactions et les échanges vivants. » (Gadet et al., 2012 : 41)

16 À partir de là Gadet et al. définissent trois niveaux écologiques: le niveau macroécologique, les écologies intermédiaires et l'écologie du discours (micro-niveau). Si ce positionnement écologique, chez Gadet et al., concerne questionne directement la vision de la langue on peut le prolonger au niveau du corpus qui devra rendre compte de cette langue.

La vision environnementale de l'analyse du discours numérique telle que la définit MarieAnne Paveau permet d'intégrer la relation d'interdépendance existant entre le texte et le contexte technologique dans les environnements numériques:

«La notion d'environnement est utilisée dans une approche des discours qui adopte une perspective postdualiste (approche externaliste remettant en cause la division mind/body et postulant que la conscience humaine se manifeste à l'extérieur de l'esprit, en particulier dans les objets et les techniques), une vision symétrique des matières langagières (la matière langagière est composite, constituée d'un assemblage entre langagier et non langagier) et une approche écologique de la production des énoncés (l'objet d'analyse n'est plus seulement l'énoncé mais l'ensemble $\mathrm{du}$ système dans lequel il est produit). Utiliser la notion d'environnement suppose de sortir d'une conception egocentrée des discours (la production des énoncés est le fait des locuteurs) pour adopter une approche symétrique distribuée (les agents producteurs des énoncés sont distribués dans l'ensemble de l'environnement). »(Paveau 2015 : En ligne)

Pourtant il semble complexe d'envisager un corpus qui puisse prendre en compte le discours selon ces perspectives écologiques, c'est-à-dire en respectant les caractéristiques incompatibles avec un figement ou une limitation des données que sont : l'instabilité, la mixité et l'incomplétude.

19 L'instabilité est amenée par l'ajout continuel de données (nouveaux «amis », nouvelles publications sur le site, publicité, nouvelles fonctionnalités du site) ainsi que par la disparition possible d'une partie des données (par exemple lors de la fermeture d'un compte, de la modification d'une publication ou du retrait de la liste d'amis).

La mixité est une propriété essentielle des données nativement numériques qui peuvent être multimodales (textes, son, vidéo, etc.), polysémiotiques (typographies, images, couleurs, etc.), technolangagières (mots cliquables, hypertextes, etc.) et/ou interactives (chaque action numérique a un impact sur le web)

21 L'incomplétude désigne la part inaccessible à la recherche d'une partie des données numériques que nous appellerons dans cet article le corpus «idionumérique». Ces données sont liées à la personnalisation du contenu sur internet: chaque utilisateur évolue dans un environnement numérique dont une partie au moins est personnalisée en fonction de ses traces numériques. Elles peuvent également être générées par l'environnement technologique matériel par lequel l'utilisateur accède au numérique (consulter Facebook depuis une application mobile ou un navigateur internet modifie l'environnement d'apparition des données). 

"collection de données langagières qui sont sélectionnées et organisées selon des critères linguistiques explicites » (Sinclair, 1996: 4). Or, renoncer à cette instabilité c'est perdre la nature technolangagière des données pour ne conserver qu'une image de celles-ci. C'est aussi renoncer à l'interactivité que permettent ces objets de recherche en les isolant des locuteurs-scripteurs-utilisateurs et de leur écosystème numérique d'apparition.

\section{Définition du lieu de corpus}

a notion de corpus, comme nous venons de le voir, est un concept flou dans le sens où il est en perpétuelle redéfinition puisqu'il doit s'adapter de façon particulière à chaque terrain. Il existe tout de même des caractéristiques communes à tous les corpus, sousentendues dans la définition de Sinclair (1996), qui sont : l'exhaustivité, la stabilité et la circonscription. Ces caractéristiques du corpus ne peuvent pas s'appliquer aux données nativement numériques sans entraîner la perte de leur numéricité.

Christine Develotte, face à cette même problématique « La volatilité des données en ligne est en soi une première fragilité des corpus que l'on veut étudier.» (2012: 516) avait répondu en proposant la notion de « corpus multimodaux ». Cette adaptation de la notion de corpus permet d'intégrer des captures dynamiques d'écrans et de rendre compte des interactions par visio-conférence qui ne sont pas évolutives. Les échanges par visio-conférence se font en respectant une unité de temps, ce que Christine Develotte appelle la quasi-synchronie (Develotte et Mangenot 2010), qui n'existe pas dans les environnements comme les réseaux sociaux numériques (désormais RSN) par exemple.

Dans son article de 2015 "Ce qui s'écrit dans les univers numériques», Marie-Anne Paveau élabore une typologie des écrits du numérique basée sur ce que l'on pourrait appeler leur degré de numéricité. Elle cite Dacos et Mounier L'Édition électronique (2010) comme première référence de ce genre de typologie, bien que leur travail soit centré sur la description d'écrits en rapport avec l'univers de l'édition.

Marie-Anne Paveau propose de considérer trois ordres linguistiques du numérique en distinguant les textes numérisés, numériques et numériqués. Nous parlerons ici de degrés de numéricité afin d'envisager le numérique comme un continuum fluide et non compartimenté puisque les phénomènes peuvent se chevaucher.

Selon cette typologie, les documents numérisés possèdent un faible degré de numéricité. Il s'agit de textes, produits en dehors des environnements numériques, qui y sont portés via des logiciels ou en étant scannés. Les documents numériques possèdent un degré intermédiaire de numéricité. Ils sont produits dans des environnements numériques hors ligne $^{1}$, ils sont modifiables et navigables. Les documents numériqués sont ceux qui possèdent le degré de numéricité le plus élevé :

«Un document numériqué est produit nativement en ligne, sur un site, un blog ou un réseau social, tout lieu numérique accueillant de la production de discours. Il présente des traits de délinéarisation de fil du discours, d'augmentation énonciative, de technogénéricité et de plurisémioticité. » (Paveau, 2015 : 7)

Autrement dit, selon la typologie de Paveau (2015), un corpus peut être constitué de documents numérisés et/ou numériques mais il exclut les documents numériqués. Pour rendre compte de ce type de documents il est nécessaire de prendre en compte leur évolutivité, soit la dimension temporelle des données. 


\section{Proposition de critères de définition du lieu de corpus} constitué dans le cadre de notre thèse ${ }^{2}$. Toutes les données accessibles à partir de ce compte appartiennent au lieu de corpus. Ce qui comprend les publications sur le journal du compte «Ma Thèse Sdl », celles apparaissant dans le fil d'actualités ainsi que celles publiées sur les journaux des membres de la liste d'amis de « Ma Thèse Sdl ». En plus des publications il y a également les messages privés, les pokes, les likes, les éléments partagés, les publicités, les notifications, les photos de profils et de journaux, et tous les autres éléments appartenant à cet écosystème numérique.

31 À partir de l'observation de ce lieu de corpus nous avons défini 6 critères constitutifs du lieu de corpus :

multimodalité : ce critère renvoie à la nature multimodale et polysémiotique des données apparaissant dans les environnements numériques. Par exemple la même publication sur le journal de « Ma Thèse Sdl » ou sur son fil d'actualité sera accompagnée d'éléments sémiotiques différents signalant à l'utilisateur, de façon contextuelle, sa position dans l'environnement.

C'est ce que montrent les illustrations 1 et 2 ci-après :

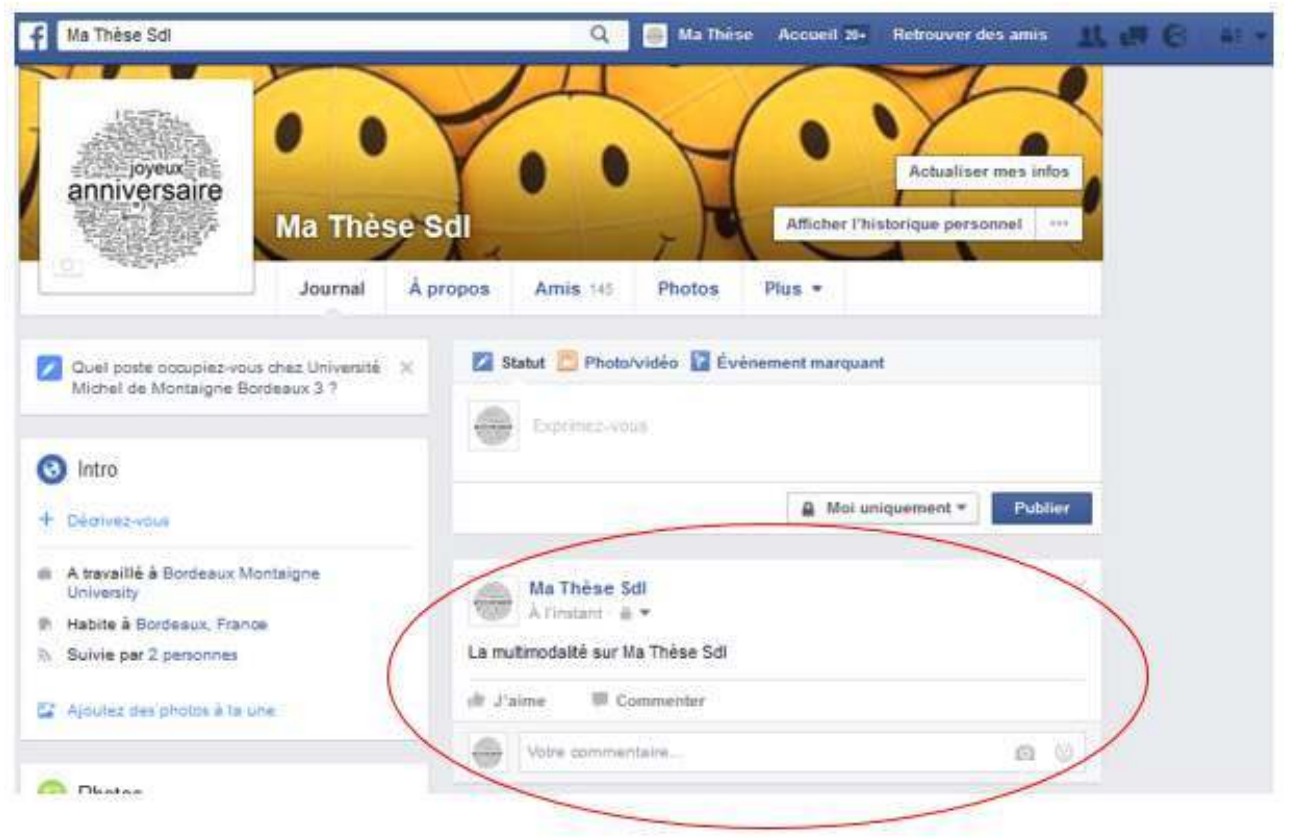




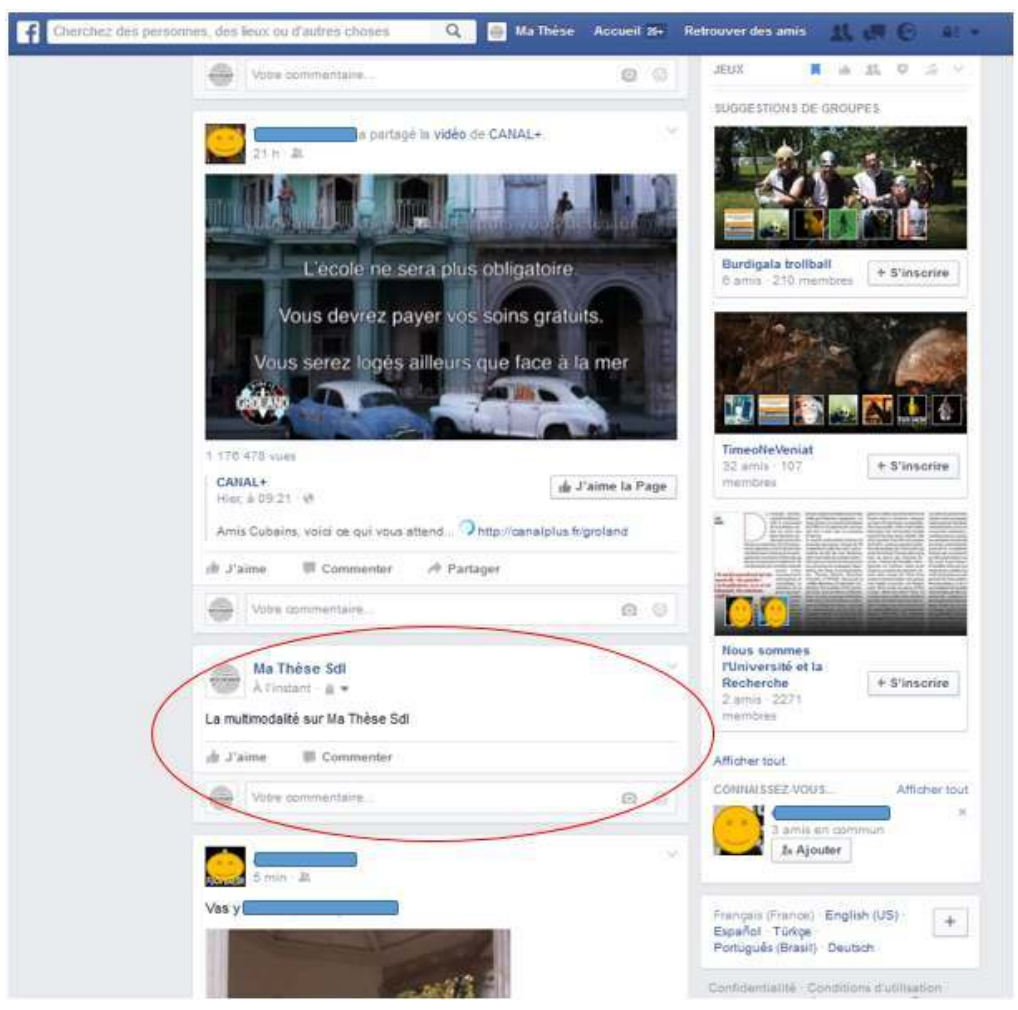

Fil d'actualité de Ma Thèse Sdl le 29/03/2016 à 13 h56 ent la physionomie du lieu de corpus. Les locuteurs ne sont pas un ensemble fixe et immuable, ils peuvent intégrer le corpus ou s'en retirer à tout moment, ce qui participe à en faire des acteurs à part entière. 
38 L'interactivité: Les locuteurs, le chercheur et l'environnement entretiennent des interactions. Ces interactions peuvent résulter d'une volonté d'impliquer les locuteurs dans la construction de la recherche, par exemple au travers de questionnaires ou de questions ouvertes. Elles peuvent également être involontaires et résulter de l'activité des locuteurs et/ou du chercheur sur le site (personnalisation du contenu, algorithmes). Elles sont également subies lorsque le site interagit directement avec ses utilisateurs (locuteurs et chercheur) par le biais des notifications.

Nous reviendrons plus précisément sur la nature des interactions et leurs implications lorsque nous présenterons les acteurs du lieu de corpus.

40 L'interconnexion: Le lieu de corpus n'est pas une île. Il s'intègre dans un écosystème numérique, lui-même impliqué dans un environnement numérique plus vaste. Ma thèse Sdl est un compte du site Facebook, écosystème numérique qui est en lien avec d'autres (Twitter, Instagram, Youtube, etc.) dans l'environnement des RSN et qui peut également créer des liens (hyperliens) avec d'autres environnements du web (sites, blogs, forums, etc...).

41 L'idionuméricité : Il s'agit de la présence, sur tous les supports numériques, de données personnalisées en fonction des traces numériques de l'utilisateur ou de la technologie qu'il utilise pour se connecter. C'est une partie du lieu de corpus qui est inaccessible pour l'instant mais qui ne doit pas être ignorée pour autant puisqu'elle représente un biais pour la recherche.

\section{Définition du corpus idionumérique}

Lorsque l'on prend pour terrain de recherche les environnements numériques on a conscience, de façon plus ou moins forte, de la nature aléatoire de la présentation des données, voire des données elles-mêmes. On sait bien, par exemple, qu'un utilisateur ne verra pas les mêmes éléments s'afficher s'il consulte Facebook à partir d'un ordinateur fixe ou s'il le fait via l'application de son téléphone mobile.

Par exemple dans le cadre du lieu de corpus «Ma Thèse Sdl» il est nécessaire de l'identifier avec le compte Ma Thèse Sdl pour avoir accès à l'ensemble des données. Sur Facebook chaque locuteur reçoit un contenu différent sur sa timeline $e^{4}$. Celle-ci est composée de publications qui sont produites et reçues dans l'écosystème auquel appartient le compte de chaque utilisateur à un moment donné 5 .

Le contenu est personnalisé automatiquement par un algorithme qui sélectionne les messages à afficher en priorité. Cet algorithme est alimenté par les traces numériques de l'utilisateur sur le site comme le nombre de clics, de likes ou de commentaires. Les icônes signalant les activités des membres de la liste d'amis (connexion au chat, brèves actualités) sont également différentes en fonction du moment auquel ils sont consultés. Mais le locuteur peut également agir lui-même sur cette personnalisation en demandant par exemple à ne plus afficher les publications provenant de certains des membres de sa liste d'amis. L'intégration de publicités, de publications sponsorisées ${ }^{6}$ ou de suggestions peut également contribuer à générer un contenu unique et éphémère. 

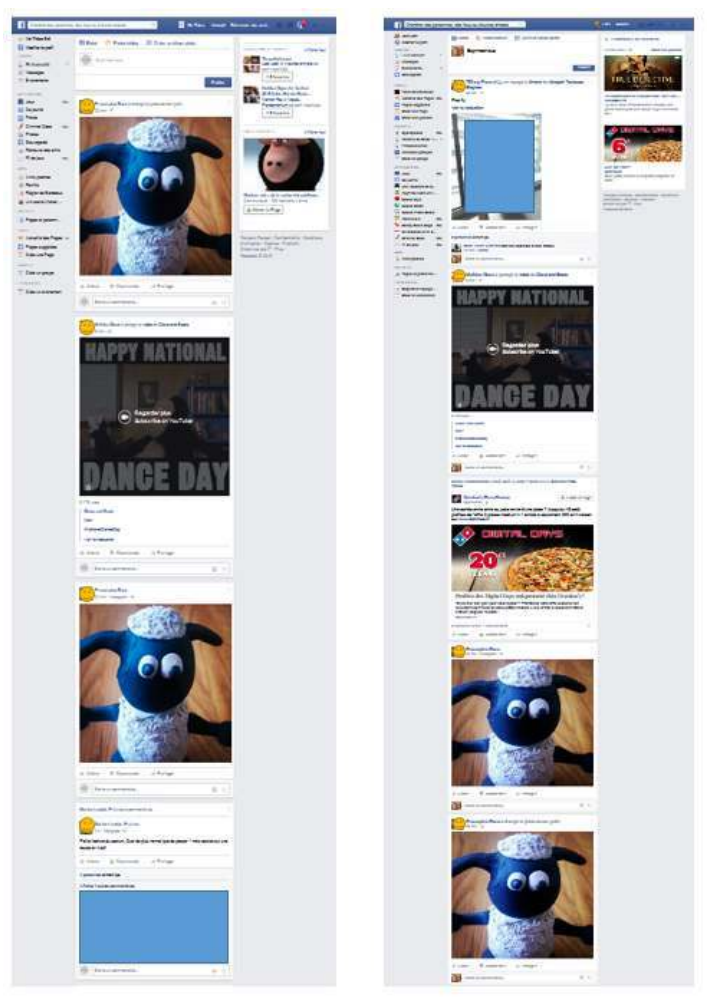

Comparaison de deux timelines Facebook capturées le 25/07/2015

Dans l'illustration ci-avant on voit deux timelines, celle de « Ma Thèse Sdl » (à gauche) et celle d'un autre utilisateur (à droite), capturées le même jour. Trois publications sur quatre sont identiques ; pourtant, leur répartition est différente et le contenu dans son ensemble change. On voit également que l'environnement de la timeline est différent en fonction du paramétrage de l'utilisateur qui le regarde.

Les éléments du profil et les raccourcis du bandeau droit peuvent être réorganisés par les utilisateurs. En répondant aux questionnaires proposés régulièrement par le site, ils peuvent également participer à la personnalisation de leur contenu. Sur d'autres réseaux sociaux numériques (c'est le cas de Twitter) il est possible de pousser plus loin la personnalisation en choisissant les couleurs du tableau de bord par exemple.

Finalement on peut dire que le compte avec lequel on accède à un contenu détermine à la fois la nature de ce contenu mais également sa présentation. Le moment durant lequel on accède à un contenu est également déterminant puisque toutes ces données sont renouvelées à chaque connexion et alimentées (à la fois en contenu mais aussi en priorité d'affichage) par les actions de l'utilisateur et des membres de son réseau.

8 La configuration générale et les paramétrages particuliers d'un site de réseau social comme Facebook entrainent une personnalisation très importante du contenu proposé aux utilisateurs. Cette individualisation peut être le fait de l'utilisateur et/ou du site et peut être conditionnée par des paramètres numériques ${ }^{7}$ et/ou technologiques ${ }^{8}$.

Cette forte personnalisation a peut-être un rôle dans la construction du discours même s'il semble complexe de le mesurer. Elle modifie les contextes de rédaction et de réception des publications sur les $\mathrm{RSN}^{9}$ et à ce titre elle doit être prise en compte et apparaitre, ne serait-ce qu'en creux, lors de la présentation de données numériques. 

Il est constitué de l'environnement sémiotique, en ligne et hors ligne, numérique et technologique, qui apparait au locuteur lorsqu'il produit une publication ou lorsqu'il la reçoit. C'est la raison qui nous a poussé à choisir le terme de "corpus idionumérique " pour référer à ces données inaccessibles mais omniprésentes.

technorelationnelle » (Paveau 2013). Le discours dans les réseaux sociaux numériques est dépendant de la médiation technologique et du réseau de relations dans lequel il est produit et reçu. Cette notion de contexte technorelationnel s'applique tout particulièrement aux réseaux sociaux sur lesquels le contexte de production et de réception du discours est différent en fonction de la timeline. Tandis que sur des sites internet ou sur des blogs le contexte de réception peut être le même pour tous les récepteurs.

Si ce niveau n'est pas accessible pour l'analyse il semble important de signaler son existence, avant peut-être, un jour, de parvenir à l'atteindre.

\section{Représentation du lieu de corpus}

53 Comment représenter un lieu de corpus qui n'a ni début ni fin par définition et dont les modifications à venir seront répercutées sur les données choisies? Faut-il limiter la représentation du lieu de corpus aux extractions que l'on peut en faire? L'extraction n'est pas le lieu de corpus, l'extraction est un corpus, elle est figée, il s'agit d'une représentation d'une sélection d'éléments à un moment $\mathrm{M}$.

Dans une perspective de recontextualisation des données et des corpus, Bénédicte Bommier-Pincemin (1999: 145) propose de considérer un « emboitement » des corpus :

«(i) un corpus existant, correspondant aux textes accessibles dont il peut disposer, (ii) un corpus de référence, constituant le contexte global de l'analyse, ayant le statut de référentiel représentatif, et par rapport auquel se calcule la valeur de paramètres (pondérations...) et se construit l'interprétation des résultats, (iii) un corpus de travail, ensemble des textes pour lesquels on veut obtenir une caractérisation, et le cas échéant (iv) un corpus d'élection, sous-corpus du corpus de travail, contrasté par rapport à celui-ci.»

Chaque corpus ((i), (ii), (iii), ...) étant inclus dans le précédent. Cette notion d'emboitement des corpus est intéressante car elle permet de considérer les corpus comme appartenant à un ensemble cohérent. Toutefois, le « corpus existant » décrit par Bommier-Pincemin ne peut pas correspondre au lieu de corpus tel que nous l'avons décrit ci-avant puisqu'il ne prend pas en compte l'évolutivité inhérente à cet objet. Pour représenter les lieux de corpus numériques nous proposons d'adopter une modélisation hiérarchisée : la représentation arborescente.

56 Comme le souligne Bommier-Pincemin, il est nécessaire de procéder à des extractions et à des spécifications des corpus. Le lieu de corpus contient un trop grand nombre d'éléments, et des éléments de natures trop différentes pour être appréhendés ensemble. Toutefois, chaque corpus doit pouvoir être replacé dans le contexte global du lieu de corpus. Nous allons le voir, la représentation arborescente permet une «traçabilité » en quelque sorte, qui ne remplace pas une restitution des données réelles mais permet de remonter à leur environnement numérique d'origine. 

telle qu'elle apparaît dans l'environnement numérique, avec les différents niveaux
d'extractions réalisés. Cette présentation vise également à rendre visibles et accessibles
les différents filtres nécessaires à l'établissement d'un outil de travail stable.

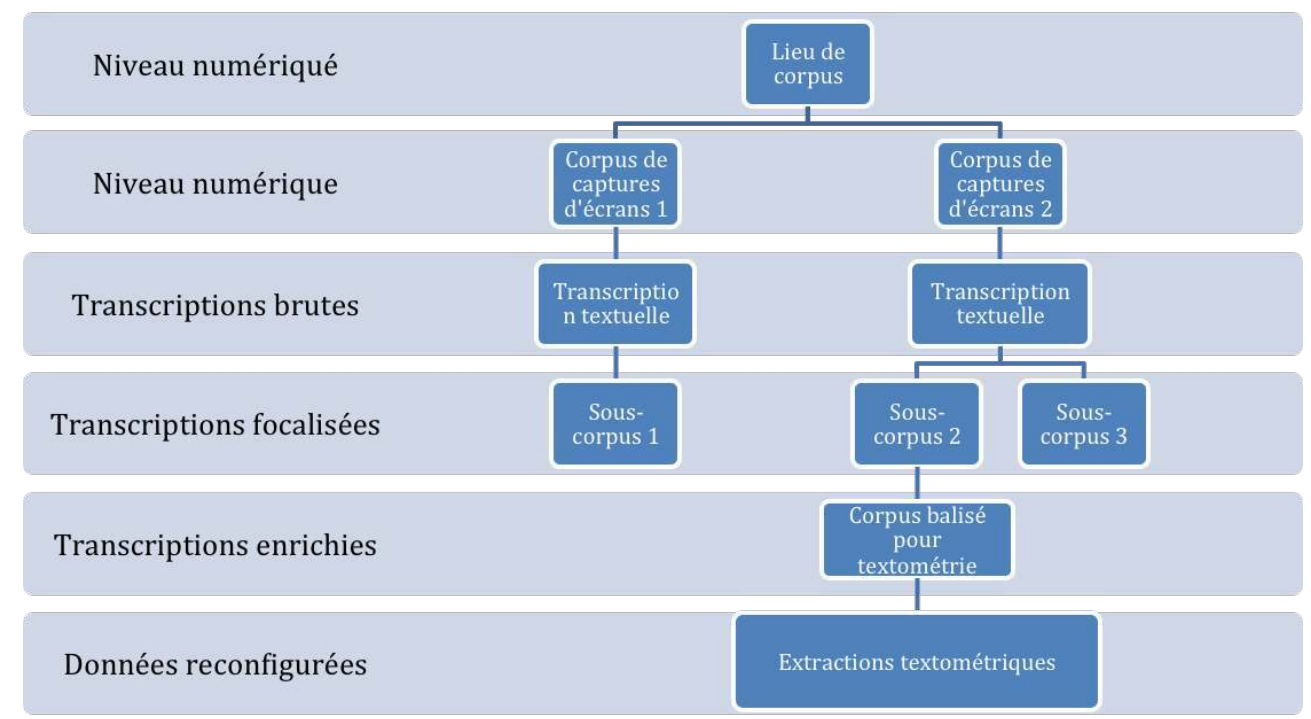

Représentation arborescente du lieu de corpus

La matrice présentée dans le diagramme ci-avant compte 6 niveaux, répondant aux besoins rencontrés pour représenter le lieu de corpus « Ma Thèse Sdl » mais on peut en imaginer autant que nécessaire. De même, chaque niveau peut supporter un nombre ouvert de sous-catégories. Nous reviendrons en détail sur chaque niveau de cette modélisation dans la suite de l'article.

Il serait possible aujourd'hui, en s'appuyant sur des logiciels de textométrie qui permettent moyennant une programmation adaptée, de conserver un très grand nombre de données et de n'en rendre visible qu'une partie à la fois. Cette technique permettrait d'arrêter l'arborescence au troisième niveau mais elle demande une grande maitrise des outils de textométrie et un temps de réalisation considérable. La subdivision actuelle du diagramme permet de pouvoir facilement aller consulter les données dans leur environnement d'apparition.

Représenter un lieu de corpus représente un enjeu complexe en l'état actuel des possibilités technologiques et méthodologiques en Sciences du Langage. La représentation arborescente est une proposition qui comporte des limites mais qui a l'avantage de prendre en compte la nature polymorphe du lieu de corpus. Nous n'avons pas trouvé pour l'instant de solution pour intégrer directement les éléments numériqués (Paveau 2015) du lieu de corpus en raison du support de présentation imposé dans le cadre du développement de cet outil ${ }^{10}$ et de la nature instable de ce type de données. Il est toutefois envisageable de rendre cette matrice dynamique et d'y intégrer une partie des éléments sous forme de liens hypertextuels en la créant directement en ligne. 


\section{Description des différents niveaux de la représentation arborescente}

\section{Niveau 1 : Données numériquées}

61 Ce premier niveau est celui du lieu de corpus, il contient des données numériquées au sens de Paveau (2015). Ce niveau de corpus est consultable uniquement en ligne, dans son environnement numérique d'apparition. Il est polymorphe, polysémiotique, évolutif et gigantesque.

Si l'on prend l'exemple d'un profil facebook il faut considérer que le niveau numériqué comprend toutes les données auxquelles ce profil donne accès. C'est-à-dire les données apparaissant directement sur les pages appartenant au profil mais également celles auxquelles ses liens d'amitiés (pour reprendre la terminologie de Facebook) lui permettent d'accéder ${ }^{11}$.

Ces données seront intégrées à des configurations sémiotiques différentes en fonction de l'environnement technologique (tablette, smartphone, ordinateur) ou numérique (depuis le journal ou le fil d'actualité) dans lequel elles seront consultées par l'utilisateur. Certaines données ne perdureront pas sous leur forme d'apparition dans ce niveau de corpus. Une partie des éléments sémiotiques sont différents à chaque consultation (publicités, suggestions, bandeau gauche fixe). Le fil d'actualité s'enrichit en permanence et il peut être compliqué de remonter jusqu'à une publication passé un délai de quelques jours. Enfin, les modifications qui sont apportées aux pseudonymes ou aux publications sur le site possèdent un caractère rétroactif.

La multimodalité, l'instabilité et le volume des données telles qu'elles apparaissent dans le lieu de corpus empêchent leur analyse puisqu'il est impossible de les représenter. C'est la raison pour laquelle la modélisation arborescente propose de subordonner au lieu de corpus différents niveaux auxquels correspondent un ou plusieurs corpus, représentant des degrés de figement et d'extraction de plus en plus importants. Toutefois, même s'il reste hors d'atteinte de l'analyse ${ }^{12}$, le niveau numériqué doit être consultable et représenté dans l'arborescence puisqu'il en est la source.

\section{Niveau 2 : Corpus numériques}

Un document numériqué est rendu numérique par un processus de figement. Il reste navigable et éventuellement consultable en ligne (Paveau 2015). Un corpus numérique est un document numérique contenant des représentations (captures d'écrans) de parties choisies dans le lieu de corpus.

Ce deuxième niveau est composé d'un instantané du niveau numériqué, pris à un moment $\mathrm{M}$ et focalisé sur un ou des phénomène(s) précis. À partir de cette représentation figée, qui reste encore difficilement exploitable en raison du très grand nombre de données qu'elle contient, on peut extraire différents sous-corpus. Chacun de ces corpus " partiels " se focalise sur un objet donné en fonction de la problématique choisie. C'est à partir de ce niveau que l'anonymisation des données peut être effectuée.

67 À partir du lieu de corpus Ma Thèse Sdl nous avons créé deux corpus numériques. Le premier focalisé sur les souhaits d'anniversaires et le second sur les pseudonymes. Pour le corpus de souhaits une période d'une année (2013) et un contexte d'apparition (les 
journaux des membres de la liste d'amis de « Ma Thèse $S \mathrm{dl} \aleph^{13}$ ) avaient été déterminés. Les captures d'écrans ont toutes été réalisées entre le $1^{\mathrm{er}}$ et le 17 janvier 2014, une fois la période observée révolue. Afin de travailler avec une certaine uniformité des affordances de l'environnement (qui propose des mises à jour régulières, surtout concernant l'archivage des données sur le site), cela a également joué un rôle sur le nombre de contacts concernés. En effet, le profil « Ma Thèse Sdl » avait 100 « amis » en janvier 2014 contre 137 en juillet 2015 et 141 en mars 2016.

Ce niveau est composé de fichiers Word, un document par « ami », contenant les captures d'écran. Seul le contexte numérique proche est conservé car il est commun à tous les interlocuteurs. On se situe au niveau de la publication et non à celle du site. Certains éléments disparaissent comme la vidéo, mais restent identifiables par une sémiotique particulière.

L'illustration ci-après est un exemple tiré du corpus de souhaits. La vidéo est identifiable par le symbole conventionnel du triangle dans un carré (qui permet de la déclencher en ligne). On retrouve également le lien hypertextuel en bleu (inactif dans cette version) ainsi que l'adresse du site à partir de laquelle elle est partagée.

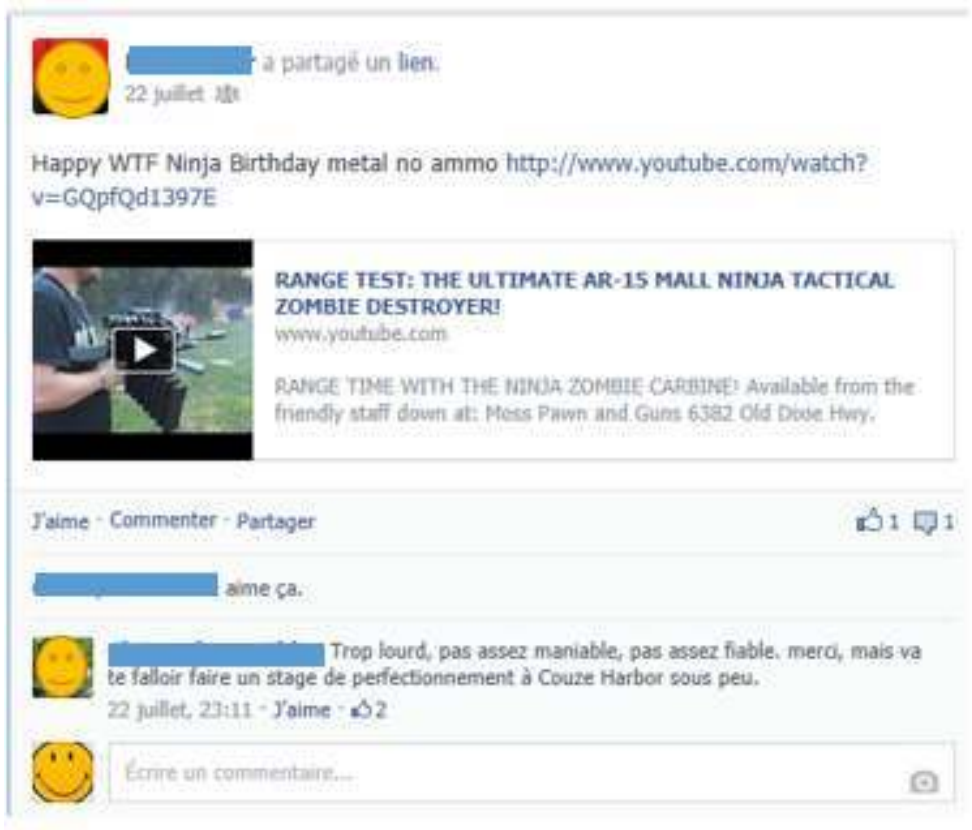

Capture d'écran niveau 2

C'est également le niveau de l'anonymisation puisque celle-ci était impossible dans le lieu de corpus. La question de l'anonymisation des données dépend de l'environnement numérique dans lequel s'intègre le lieu de corpus. Dans le cas de Ma Thèse Sdl, Facebook étant un réseau social semi-privé, l'anonymisation était nécessaire pour présenter les données dans le cadre d'une recherche scientifique et publique.

71 Cette représentation figée du corpus permet de le restreindre et de stabiliser les données. Il s'agit d'une archive qui fournit une base de travail stable et immuable impossible à retrouver en ligne et sans laquelle un travail de recherche sur le long terme semble impossible. 
Malgré le figement, ce niveau conserve un grand nombre de données environnementales. On le voit dans l'illustration 2 , au-delà des éléments sémiotiques liés à la présence d'une vidéo, de nombreux éléments discursifs et méta-discursifs sont présents :

La photo de profil et le pseudonyme,

La mention « a partagé un lien »,

La date de publication

Le symbole représentant le réglage des paramètres de confidentialité appliqués à cette publication par le locuteur-scripteur-utilisateur qui la publie.

Le texte accompagné de l'adresse URL puisqu'il s'agit du partage d'un contenu venant d'un autre site ${ }^{14}$

Le contenu partagé (ici la vidéo)

Les trois boutons (mots cliquables) de la publication Facebook : «j'aime », " Commenter », «Partager ». Suivi de la représentation symbolique du nombre de likes et du nombre de commentaires

Les pseudonymes des locuteurs-scripteurs-utilisateurs likeurs accompagné d'un énoncé de commentaire généré automatiquement par le site

La photo de profil, le pseudonyme et l'énoncé du commentaire

La date, l'heure, le bouton «j'aime » et le symbole du nombre de likes attribués au commentaire.

La photo de profil de l'utilisateur qui consulte cette publication suivie de la fenêtre de commentaire.

On peut également compter la présentation sémiotique de la publication qui est reproduite ici. En particulier les couleurs qui ont de l'importance dans cet environnement numérique puisqu'elles désignent les formes cliquables (liens, symboles, mots). Elles permettent également de séparer les zones de contenu, fond blanc pour la publication, fond gris pour les commentaires et de nouveau un fond blanc pour la zone de texte.

\section{Niveaux 3 et 4 : Transcriptions brutes et focalisées}

85 Si le deuxième niveau contient des corpus, les niveaux suivants servent à décomposer ces corpus en plusieurs sous-corpus focalisés.

86 Le troisième niveau consiste en une transcription brute des données du deuxième. À partir de ce niveau, les données sont essentiellement de nature textuelle. À chaque corpus de second niveau ne peut correspondre qu'une seule entrée au troisième niveau. Il s'agit de ne transcrire que les données brutes, sans filtre d'analyse qui pourrait générer différents sous-corpus.

87 Les images et les couleurs disparaissent, en revanche plusieurs informations contextuelles restent. Certaines métadonnées sont conservées à ce niveau comme les dates, heures, likes ainsi que le nombre de commentaires. À partir de ce niveau, le corpus peut être analysé de façon «traditionnelle » en linguistique puisqu'il est composé d'une liste d'énoncés.

Si l'on reprend l'exemple de l'illustration «capture d'écran niveau 2 », la version apparaissant au niveau 3 est la suivante : 


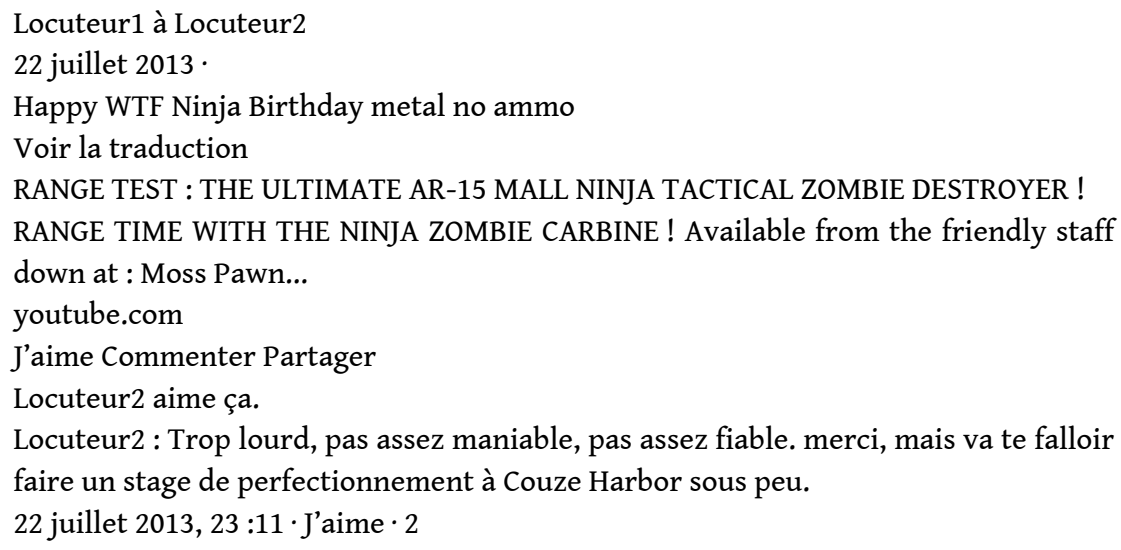

Dans l'exemple ci-dessus, pour simplifier la lecture, le souhait d'anniversaire est surligné en jaune et le commentaire en bleu. Lors de la phase de traitement des fichiers, cette convention permet de repérer plus rapidement les éléments rédigés par les utilisateurs.

On peut voir dans cet exemple la perte de référence des éléments technolangagiers qui semblent parasiter le discours des locuteurs, en tout cas leur perception hors contexte. Cet appauvrissement justifie en partie l'existence du troisième niveau, à partir duquel on entre dans des extractions utiles lors du travail de recherche mais non représentative du corpus.

91 Le quatrième niveau est le prolongement du troisième à partir duquel seuls quelques éléments sont répartis en sous-catégories. À partir de l'exemple représenté dans l'illustration "capture d'écran niveau 2 " nous avons créé un sous-corpus regroupant tous les énoncés constituant des souhaits d'anniversaires et un autre contenant tous les commentaires. Chaque sous-corpus est composé de fichiers Word (1 par ami du profil "Ma Thèse Sdl»). Il serait également possible de constituer les sous-corpus «métadonnées » ou « technoformes », en fonction de la focalisation des recherches faites sur le corpus.

Voici un aperçu de la transcription de la publication précédente au quatrième niveau :

Fichier des souhaits

Locuteur1 : Happy WTF Ninja Birthday metal no ammo

Fichier des commentaires

Locuteur2 : Trop lourd, pas assez maniable, pas assez fiable. merci, mais va te falloir

faire un stage de perfectionnement à Couze Harbor sous peu.

93 Les niveaux 3 et 4 sont les derniers à contenir des données naturelles, bien que totalement décontextualisées. Toutes les opérations de filtrage ont été réalisées. Dans les niveaux suivants, un travail susceptible de modifier en partie les données sera effectué.

\section{Niveau 5 : Transcriptions enrichies}

94 L'enrichissement concerne essentiellement la constitution de corpus "préparés " pour les logiciels de TAL ${ }^{15}$ (textométrie ou autre). Certains logiciels de textométrie demandent d'ajouter des balises à l'intérieur du texte. C'est le cas d'Iramuteq ${ }^{16}$ et de $\mathrm{TXM}^{17} \mathrm{par}$ exemple, qui demandent un travail différent en fonction des choix d'importation des données à l'intérieur du logiciel.

Le corpus de souhaits d'anniversaires sur Facebook a été préparé pour être analysé par $\mathrm{TXM}^{18}$. Cette préparation impliquait la création d'un dossier contenant les versions en 
texte brut extraites du niveau 4. Dans l'illustration suivante les données sont préparées pour être traitées par le logiciel de textométrie.

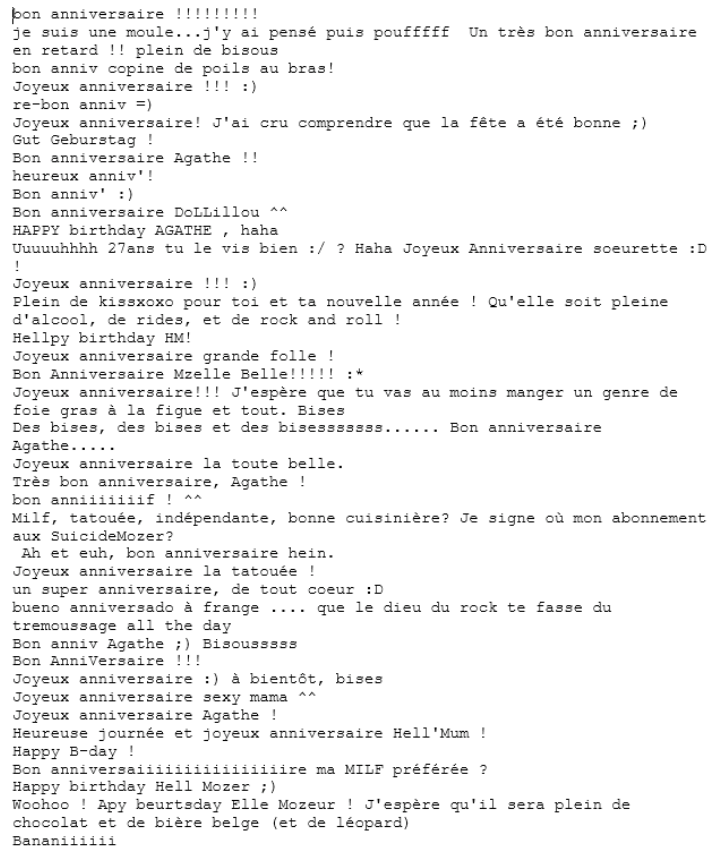

\section{Fichier texte brut souhaits d'anniversaire}

TXM ne demande pas beaucoup de préparation des données, mais à partir de ce fichier il est possible d'introduire des balises pour intégrer des métadonnées supplémentaires. Pour d'autres logiciels (ou une utilisation plus avancée de TXM) il est nécessaire d'ajouter beaucoup plus d'informations à l'intérieur des fichiers, il faut parfois créer des dossiers de métadonnées à part.

\section{Niveau 6 : Données reconfigurées}

97 Le sixième niveau est constitué de données pré-analysées de façon automatique. Il peut s'agir de sélections d'énoncés dans le but de composer un exemplier représentatif du corpus lorsqu'il y a une grande quantité de données. C'est également à ce niveau qu'il est possible de classer les extractions provenant des logiciels de textométrie.
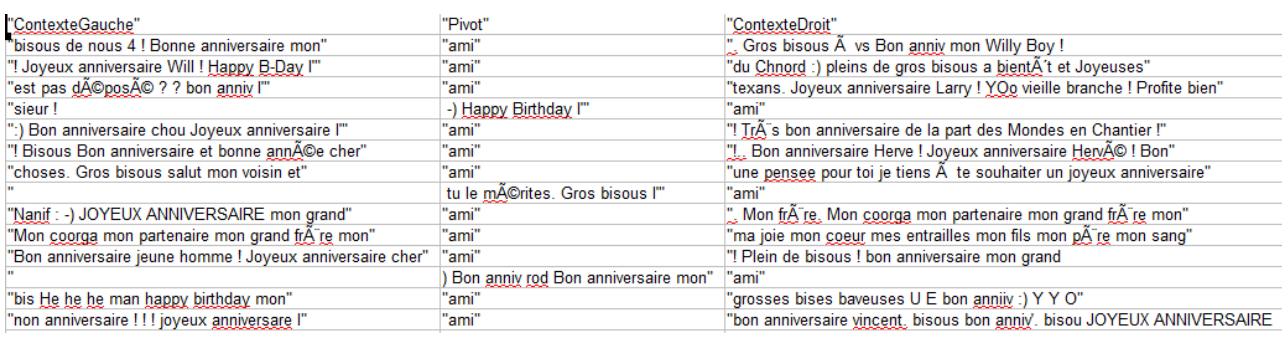


\section{Extraction TXM} logiciel.

L'illustration ci-avant montre une extraction provenant de TXM sur laquelle on voit apparaitre les données organisées autour d'un mot pivot prédéfini. Il s'agit toujours d'extractions de travail, c'est pourquoi elles sont intégrées au corpus, mais elles sont déjà passées au filtre de l'analyse quantitative. Comme le montre la différence entre les deux dernières illustrations les données sont les mêmes mais elles ont été reconfigurées par le

Le sixième niveau est le dernier que représenté dans cette arborescence, mais, chaque corpus étant différent on pourrait imaginer bien d'autres configurations à partir de la même matrice hiérarchisée. L'intérêt de cette méthode est de permettre à la fois la traçabilité et la pérennité des données. Ainsi que l'exploitation maximale du corpus, dans différentes temporalités.

\section{La place des acteurs dans le lieu de corpus}

L'utilisation d'un lieu de corpus, au-delà d'interroger sur sa représentation, demande également un questionnement et un positionnement par rapport aux différents acteurs qui le constituent. Nous avons identifié trois acteurs du lieu de corpus: les utilisateurs, l'écosystème numérique d'apparition et le chercheur. Du dialogue entre le chercheur, les locuteurs et le site, naitra la mise en matière du corpus.

Le positionnement du corpus, en tant que lieu non fini et interactif, a un impact concret sur celui du chercheur. Travailler «sur» le discours numérique demande de travailler « dans » les environnements numériques. Leur connaissance est un savoir qui s'acquiert par la pratique. Quelle est la posture du chercheur dans ce contexte?

Pour comprendre les relations entre les trois acteurs du corpus il faut accepter que la fonction d'utilisateur soit commune aux locuteurs et au chercheur. L'utilisateur est celui qui utilise les outils numériques et qui évolue dans les environnements numériques. On peut être utilisateur sans être scripteur. La rédaction n'est pas la seule action permise par les environnements numériques par exemple si on utilise le poke ou le like sur Facebook, on ne rédige rien pourtant on utilise et on communique. L'inverse n'est pas vrai puisqu'il est nécessaire d'utiliser les fonctionnalités des environnements numériques pour rédiger un message. Il faut également être utilisateur pour consulter les environnements numériques, puisque se connecter c'est déjà utiliser.

Il n'existe pas de manuel clé en main des environnements numériques ; même si on peut trouver des dictionnaires et des tutoriels en ligne ceux-ci sont continuellement dépassés par l'évolution de la technologie et des pratiques des utilisateurs. Les environnements numériques évoluent grâce à des mises à jour des logiciels mais également au travers de l'utilisation qui en est faite par les internautes qui peuvent détourner ou créer des outils de communication. C'est notamment le cas du hashtag qui a été créé et utilisé en masse par les utilisateurs de Twitter, ce qui a poussé le site à l'intégrer à ses fonctionnalités.

4 À partir de ce positionnement, nous proposons de considérer que les locuteurs et le chercheur ont en commun la fonction d'utilisateur. Nous parlerons donc de locuteursutilisateurs et de chercheur-utilisateur. 


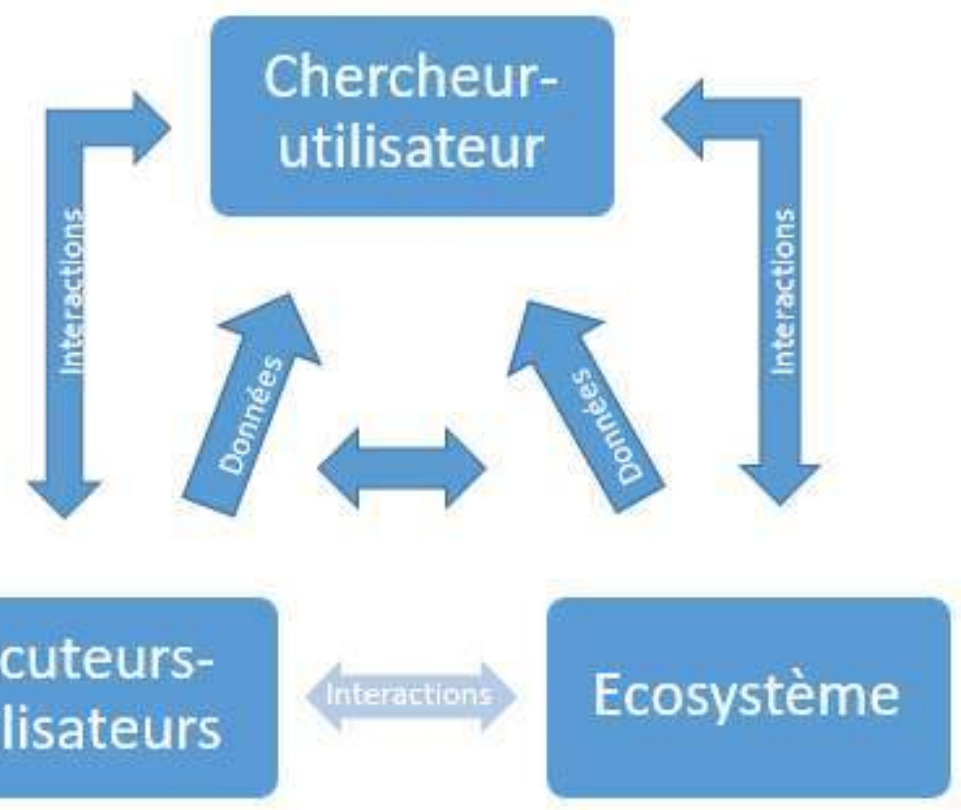

Relations entre les acteurs du lieu de corpus

C'est parce que le chercheur est un utilisateur qu'il entre en interaction avec les autres utilisateurs et l'écosystème numérique. Cela implique que le chercheur ait également un impact sur l'écosystème ainsi que sur les autres utilisateurs, comme on le voit dans la figure ci-avant. Pour conserver une position de chercheur il est donc important que celuici ait conscience de sa position d'utilisateur. Cette question de l'implication du chercheur dans sa recherche a déjà été abordée en sociolinguistique du point de vue de la subjectivité :

«En sociolinguistique, la double question de la subjectivité / objectivité, et de la neutralité / implication est notamment soulevée par des linguistes travaillant sur des situations de minorations linguistiques dont ils se revendiquent « natifs ». Pour ces linguistes, les dimensions «action » ou «implication » de la recherche sont d'autant plus évidentes qu'ils articulent la description de langues minorées (dont ils sont / se définissent comme locuteurs) dans leur rapport à la langue socialement dominante, à la description de conflits linguistiques auxquels, aussi bien en tant qu'acteurs sociaux qu'en tant que sociolinguistes, ils participent en même temps. » (Bretegnier, 2009: 30)

106 Dans le cas des recherches numériques il ne s'agit pas uniquement de savoir si le chercheur doit ou non «objectiver» sa recherche, il y a également une dimension d'interaction que l'on pourrait rapprocher de la recherche d'intervention telle qu'elle est pratiquée en sociologie :

« En sociologie, les chercheurs d'intervention défendent l'idée de construction et de reconnaissance d'une recherche admettant en son champ l'intervention sur et avec le terrain, et s'interrogeant sur la manière dont cette inter-action, ce « venir-entre ", l'amène à son tour à se redéfinir. » (Bretegnier, 2009 : 32)

Le choix de travailler à partir et dans un lieu de corpus et plus globalement le fait de situer ses recherches dans les environnements numériques, demande au linguiste de puiser des outils théoriques et méthodologiques dans les autres sciences humaines.

«L'une des questions cruciales pour l'avenir sera précisément de s'interroger sur les pratiques pertinentes qui nous aidera à comprendre cette réalité que nous 
contribuons à produire tout en accompagnant les acteurs de terrain. Ceci appelle un débat tant entre les sociologues s'intéressant au thème de l'intervention qu'au sein même de la discipline, car, dans cette optique, la science ne naît pas d'une prise de distance, elle se dégage à travers un mouvement constant de va-et-vient entre le savant et son objet. » (Vrancken, 2001 : 261)

Les interrogations de Vrancken, on le voit, sont tout à fait valables dans le cadre de la construction d'un lieu de corpus. On pourrait comparer ce qui se passe dans le lieu de corpus à une colocation. Un bail tacite relie le chercheur-utilisateur, les locuteursutilisateurs et l'écosystème. Cette métaphore de la collocation permet de donner une place à la technologie qui est partie prenante de l'environnement et du discours qui y est produit.

\section{Les locuteurs-utilisateurs}

Ils agissent sur l'écosystème numérique en produisant des publications et en agissant sur le site (likes, nombre de vidéos vues, etc.) ce qui alimente les algorithmes de personnalisation. Ils fournissent des données au chercheur de façon passive (en laissant accès à leurs publications sur le site) ou active (en participant à des enquêtes). Ils peuvent également être à l'origine d'un échange direct avec le chercheur (messagerie ou publication).

110 Les locuteurs ont une place centrale dans le corpus puisque celui-ci est constitué des énoncés qu'ils produisent. Pourtant, s'ils fournissent la matière première de la recherche, ils sont souvent écartés de la construction du corpus en tant qu'objet d'étude. Cette partie revient au linguiste qui le structure en fonction de choix méthodologiques et théoriques, auxquels les locuteurs n'ont pas accès.

Dans le lieu de corpus, les locuteurs-utilisateurs ont un rôle à jour puisqu'ils doivent donner leur consentement et le maintenir ${ }^{19}$ et ils peuvent participer à la recherche au travers d'interactions avec le chercheur. Le locuteur-utilisateur n'est pas un simple producteur de données, il devient un acteur à part entière du lieu de corpus.

112 Dans ces conditions il semble légitime de parler de "co-construction » du lieu de corpus. D'un côté, les locuteurs offrent leurs conversations, leurs traces linguistiques numériques à l'étude et peuvent participer à une discussion autour de ces productions. De l'autre, le chercheur décrit, classe et analyse en s'appuyant à la fois sur ses connaissances théoriques et méthodologiques et sur les fruits de la discussion qu'il entretient avec les locuteurs.

\section{L'écosystème numérique}

113 Il agit sur les locuteurs-utilisateurs en proposant un cadre sémiotique, en leur envoyant des notifications et en offrant des affordances. Il est le terrain d'étude du chercheur ce qui configure les données sémiotiques contextuelles du lieu de corpus.

114 La technologie doit être considérée comme un acteur à part entière du lieu de corpus. Dans la mesure où elle fait partie de l'interaction en s'adressant directement aux utilisateurs (locuteurs et chercheur) mais aussi en incitant au discours ou en le facilitant.

115 Pour filer la métaphore de la colocation il faudrait préciser la place du bailleur, ici l'écosystème numérique. Le site qui accueille le lieu de corpus joue un rôle déterminant 
dans son élaboration. C'est lui qui offre la structure nécessaire à l'établissement de ce lieu numérique dont il décide de l'agencement et des potentialités technologiques.

Autrement dit, l'écosystème numérique ne se contente pas (lui non plus) de rester un hébergeur passif. Il entretient une relation dynamique avec ses utilisateurs en proposant de nouvelles applications d'une part et en s'adaptant à leur utilisation d'autre part. Nous avons relevé trois types d'influences exercées directement ou indirectement par le site sur le corpus : technologiques, structurelles ou connectives.

Influences technologiques : on se rapproche ici de la question des affordances. L'écosystème numérique met à disposition des locuteurs des outils technologiques qui métissent leur discours et en font des matières technolangagières.

Certains outils sont décidés par le site et font partie de sa stratégie de singularisation. C'est le cas des boutons de like ou de partage sur Facebook. D'autres sont proposés par le site pour répondre à un «besoin » ou à une utilisation effective des locuteurs comme nous l'avons montré pour le hashtag et son intégration a posteriori sur Twitter.

Les matières technolangagières sont des composantes essentielles du lieu de corpus, elles sont directement conditionnées (ou influencées) par le site hébergeur. Si l'on reprend l'exemple du hashtag, sur Facebook son utilisation a également précédé son activation technologique sur le site, toutefois les énoncés contenant des hashtags ne sont devenus technolangagiers qu'à partir du moment où Facebook a permis cette fonctionnalité. Les évolutions technologiques du site ont donc un impact direct sur la matérialité des énoncés du corpus.

120 Influences structurelles: au-delà de l'aspect technologique, la structure même du site subit de régulières modifications qui peuvent intervenir à plusieurs niveaux dans le corpus.

121 Sur Facebook, les politiques du site en matière de protection de la vie privée vont vers une ouverture de plus en plus importante des paramètres de confidentialité. Indirectement, ces modifications peuvent avoir des conséquences, par exemple sur l'utilisation de pseudonymes ou la falsification de certaines informations (comme la date de naissance). Les photos de journal et de profil étant publiques par défaut, les locuteurs peuvent également être tentés de les modifier pour préserver leur anonymat.

Les évolutions structurelles ont également une influence sur les éléments sémiotiques du corpus. La composition du bandeau gauche de Facebook a, par exemple, été plusieurs fois modifiée par le site durant les trois années qu'a duré notre travail sur le lieu de corpus Ma Thèse Sdl. Intégrant petit à petit de plus en plus d'éléments du fil d'actualité avec des formes cliquables et un accès au like et au commentaire direct.

Enfin, l'influence structurelle se retrouve au niveau de l'archivage des publications sur les fils d'actualité, y compris celui de notre lieu de corpus. Les modifications successives, ayant un caractère rétroactif, ne permettent pas de retrouver les données sous le même format que lors de leur apparition.

Influences connectives: nous choisissons ici le terme "influence connective» en référence à la connectivité écologique qui permet de poursuivre la métaphore amorcée par Marie-Anne Paveau sur l'écologie numérique. La connectivité écologique est une notion d'écologie du paysage qui désigne le "fait que deux taches de même type soient adjacentes, jointes, dans l'espace » (Baudry et Burel, 1999 : 58). 
125 En ce qui concerne les environnements numériques, nous choisissons le terme de connectivité écologique pour désigner les phénomènes de publication simultanée, par un même locuteur, dans des écosystèmes différents. Les écosystèmes concernés sont alors connectés, au moment de la rédaction, par la publication.

Il est par exemple possible de lier un compte Facebook et un compte Twitter, à partir d'un paramétrage particulier sur l'un, l'autre ou les deux sites. Une fois les comptes liés toutes les publications émises sur Facebook avec un paramétrage de confidentialité ouvert (« public ») seront également publiées sur Twitter, de manière automatique.

es publications prendront la forme adaptée à leur écosystème numérique d'apparition.

Un statut sur Facebook et un tweet sur Twitter. Si la publication n'excède pas les 140 caractères (taille limite du message imposée par Twitter) il est impossible de déterminer l'écosystème de rédaction du tweet. Dans le cas où la taille de la publication est supérieure à 140 caractères (puisque c'est autorisé sur Facebook), le tweet qui en résultera sera tronqué et sera terminé par un lien renvoyant à la publication dans son écosystème de rédaction.

Toutes les fonctionnalités (liens, hashtags, etc.) sont actives et fonctionnelles à l'intérieur de leur écosystème d'apparition. Cela veut dire, dans le cas des hashtags notamment, que l'on obtient deux discours différents à partir du message d'origine, puisque la base de données n'est pas commune aux deux environnements.

\section{Le chercheur-utilisateur}

Il reçoit les données des locuteurs-utilisateurs et de l'écosystème numérique mais il entretient également, parallèlement, des interactions avec eux. Il peut interroger directement les locuteurs utilisateurs, via les outils proposés par l'environnement (messages privés, publications, etc.). Ses publications auront également un impact sur le contenu qui apparaitra sur le site pour les utilisateurs faisant partie de son réseau. De la même manière les autres utilisateurs pourront avoir des interactions avec lui.

Il aura les mêmes échanges que les autres utilisateurs avec l'écosystème. Il agira sur lui en produisant des publications et en agissant sur le site (likes, nombre de vidéos vues, etc.) ce qui alimente les algorithmes de personnalisation. Et l'environnement agira sur le chercheur-utilisateur en proposant un cadre sémiotique, en lui envoyant des notifications et en proposant des affordances.

Les enjeux de la co-construction du corpus sont importants dans l'étude du discours produit dans le cadre des RSN. Il s'agit de reconnaitre la place des locuteurs et du site afin, également, de pouvoir s'en affranchir. Nous pensons que cette remise en question est importante pour préserver une différenciation claire entre la position du chercheur et celle des autres acteurs du corpus, condition sine qua non d'une mise à distance de l'objet nécessaire à son étude scientifique.

132 Le dernier acteur-colocataire du lieu de corpus tel que nous venons de le décrire est le chercheur. Celui-ci se retrouve dans une position inédite en linguistique mais qui a déjà été débattue en sociologie, en anthropologie ou en ethnologie. Le chercheur-participant, intégré à la population étudiée doit avoir conscience de sa position ainsi que des limites et des avantages de celle-ci.

«L'observation ethnographique est toujours, selon l'expression consacrée, plus ou moins participante. Pour que l'insertion s'opère, pour que l'enquête fonctionne, il 
faut accepter d'échanger une position d'observateur extérieur contre celle de partenaire ou d'acteur au sein du groupe étudié. »

(Schwartz dans Anderson 1993 : 270)

Le lieu de corpus numérique, augmenté, enrichi, redéfini tel que nous venons de le présenter, ouvre donc sur une redéfinition de la posture du chercheur. Nous parlerons ici des postures du chercheur, au pluriel, car il ne s'agit plus d'un duo impliquant un chercheur et un corpus, mais d'une situation d'interaction comprenant plusieurs acteurs. Dans le cadre d'une observation participante de cette situation, le chercheur doit assumer plusieurs rôles, plusieurs postures, qui sont fonction des autres interactants. dans lequel il s'intègre. Nous ne prétendons pas donner ici une description exhaustive des différentes facettes et postures du chercheur qui serait valable pour tous les lieux de corpus. Il s'agit plutôt d'illustrer et d'attester, au travers de notre expérience de création $\mathrm{du}$ lieu de corpus Ma Thèse Sdl, leur existence. Nous avons dénombré trois types d'acteurs (les locuteurs, le site et le chercheur) auxquels nous faisons correspondre trois postures : l'ami, l'utilisateur et l'espion.

Posture de l' "ami » : cette posture est provoquée, dès la création du compte Facebook Ma Thèse Sdl, par le fonctionnement du réseau social et qui nécessite de passer par la demande d'ajout à la liste d'amis. Facebook met en scène des relations qu'il qualifie de «liens d'amitié»; choisir ce site comme écosystème numérique implique donc nécessairement de construire une liste d'amis.

Cet étiquetage fait par le site reste évidemment de surface. Les locuteurs ne sont pas plus dupes que nous sur la réalité de cette "amitié». Pourtant, l'utilisation de ce terme encourage à une certaine proximité qui n'aurait sans doute pas été la même dans le cadre d'entretiens en face-à-face. Il nous semble également que la notion "d'amis sur Facebook » est différente de celle « d'amis ». Ainsi des connaissances éloignées, des amis d'enfance, des collègues ou des membres de la famille peuvent tous appartenir à la catégorie des «amis Facebook», ils ne seront pourtant pas nécessairement des «amis » au sens strict du terme.

Les deux énoncés "Untel est mon ami Facebook » et "Untel est mon ami » renvoient à des liens différents. Le premier exprime le fait qu'Untel fait partie de mes contacts sur le site de réseautage et le second révèle un lien relationnel fort entre moi et Untel.

Le profil Ma Thèse Sdl entre, pour les locuteurs du corpus, dans cette catégorie un peu floue des «amis sur Facebook». Le nom choisi pour ce profil renvoie à un objet et non directement à une personne, pourtant nous avons toujours employé la première personne dans les publications faites à partir de ce compte. Pour la partie la plus active des locuteurs (ceux qui participent aux questionnaires), il y a identification entre le profil $\mathrm{Ma}$ thèse Sdl et le chercheur. 


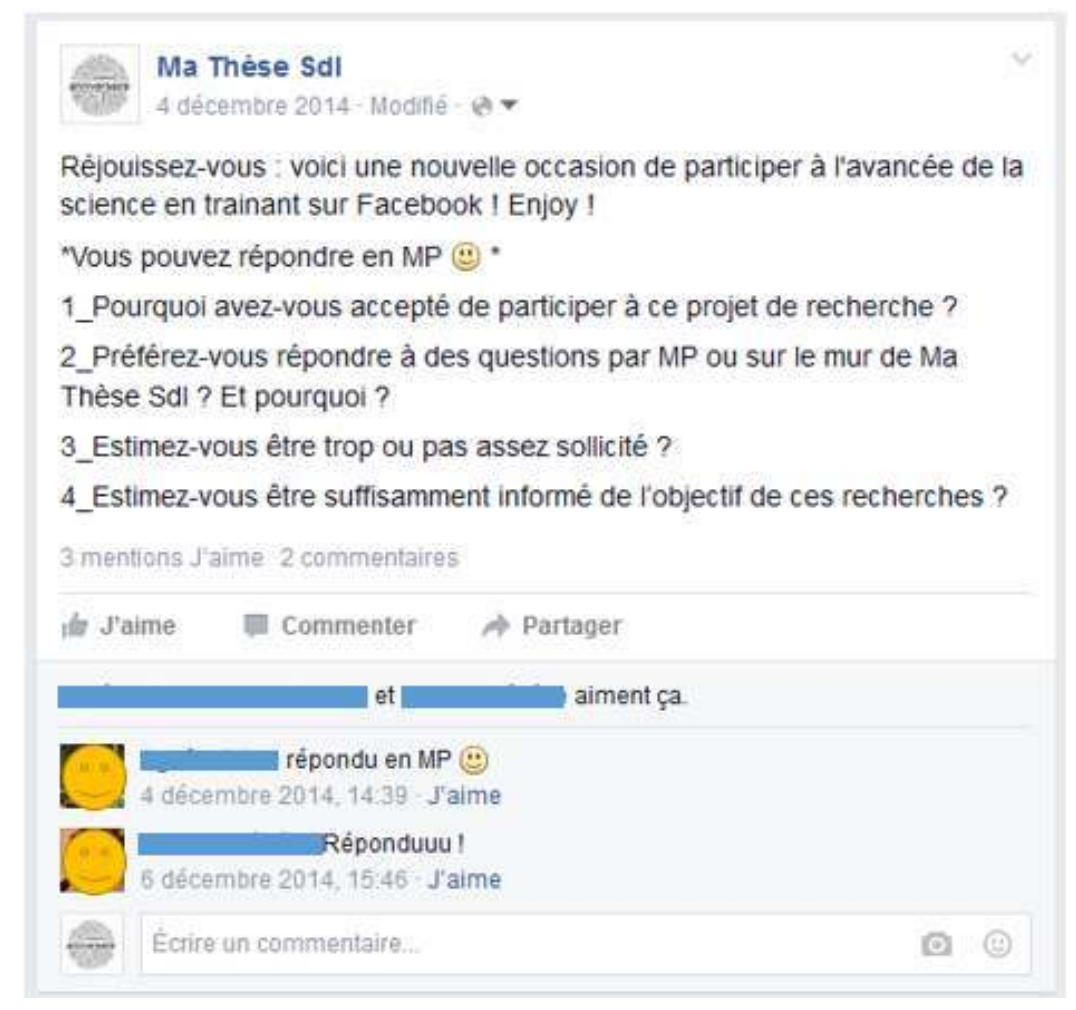

Illustration de la posture de l'ami

Le ton des publications apparaissant sur le journal de Ma thèse Sdl est informel. Les nôtres comme celles des locuteurs sont assez relâchées et contiennent souvent des notes d'humour. On le voit dans l'illustration ci-avant, l'introduction de notre message a une tournure exclamative et un vocabulaire familier («trainant», «Enjoy!»). Il contient également un émoticône qui renforce la connivence induite par le message. Les deux commentaires sont rédigés sur le même ton: pas de pronoms sujets, émoticône (en miroir) dans le premier et allongement syllabique + tournure exclamative (miroir également) dans le second.

La posture de l'ami est donc nécessaire pour entrer en contact avec les locuteurs et pour entretenir la relation. Il s'agit de jouer le jeu du réseau social numérique dans lequel on se situe, au même titre que les autres utilisateurs.

Posture de l'utilisateur du réseau : assumer la posture de l'ami demande nécessairement d'assumer celle d'utilisateur du réseau social numérique. Chaque environnement possède ses codes, ses fonctionnalités technologiques, ses applications et pour entrer en relation avec les autres membres il faut les intégrer et les utiliser.

Nous avons différencié notre compte Facebook personnel de celui du corpus, ils n'ont ni le même pseudonyme, ni la même identité sémiotique, ni les mêmes « amis » (bien qu'une partie de la liste d'amis soit commune). Cette double existence sur le réseau n'est pas cachée aux locuteurs qui répondent indifféremment aux interventions émanant de l'un ou l'autre compte.

144 Les réseaux sociaux numériques sont interconnectés et pour assumer une posture d'utilisateur d'un réseau social il faut posséder la connaissance des autres RSN et la 
pratique de certains. Les savoirs numériques sont construits dans la pratique. Il existe des guides d'utilisations et des tutoriels sur le net mais comprendre l'étendue des fonctionnalités demande de les utiliser.

Durant les trois années de notre travail de recherche nous avons découvert de nombreux RSN et nous sommes impliqués dans certains d'entre eux (comme Twitter, Instagram ou Pinterest), en fonction de nos affinités et des liens potentiels avec Facebook. Nous avons également découvert certaines fonctionnalités de Facebook dans la pratique ou suite à des notifications du site. Nous avons pris le parti de tester chaque fonctionnalité rencontrée de manière systématique. Cette méthodologie ne nous assure pas une connaissance exhaustive du site mais nous a permis de mieux comprendre son fonctionnement.

Il nous est également arrivé de consulter les "amis » de Ma Thèse Sdl lorsque nous ne comprenions pas une application. Ça a été le cas notamment pour le Poke, comme on le voit dans l'illustration suivante, sur lequel nous avons questionné les locuteurs.

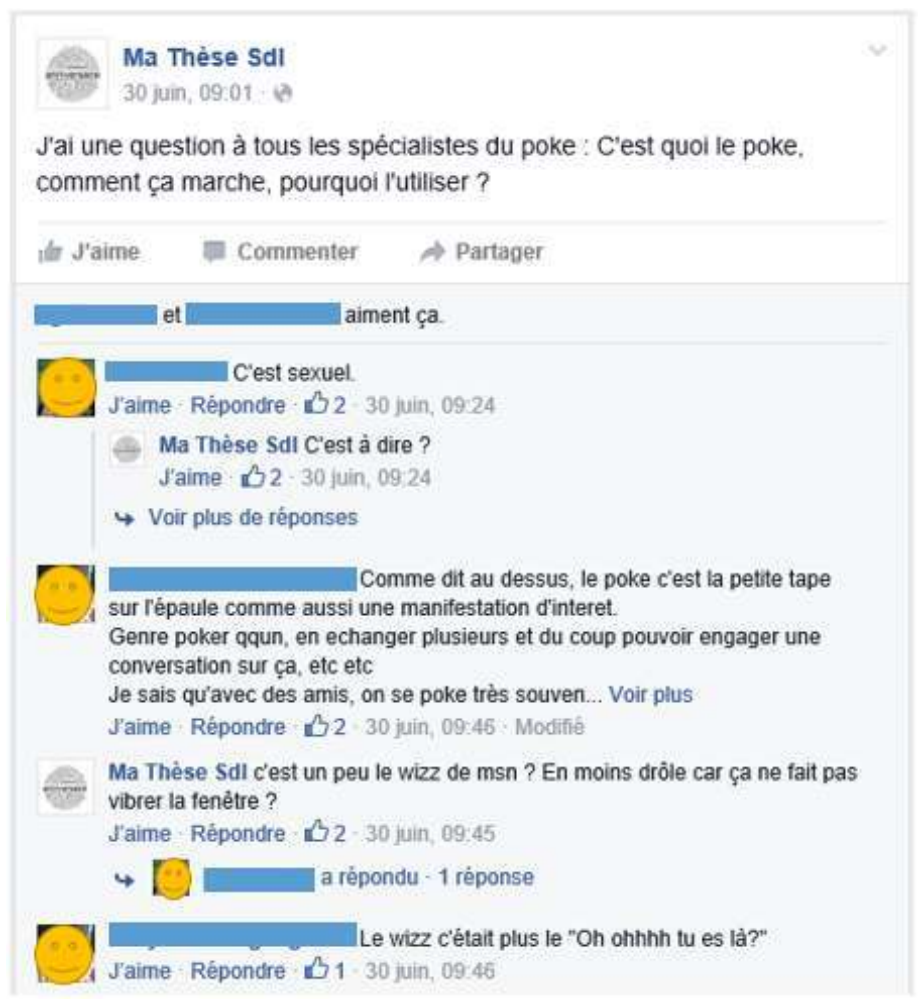

Illustration sur le Poke

Ce statut a été commenté 31 fois par les locuteurs (sans compter les nombreuses réponses aux commentaires) qui, comme on le voit dans l'extrait représenté ci-avant, ont été très pédagogues et se sont employés à définir le poke. Malgré ces explications, nous ne saisissions pas réellement la différence avec des applications antérieures. Nous avons donc testé le poke de façon systématique en deux temps : 1 / en pokant tous les amis de notre liste d'amis, 2/ en répondant systématiquement aux pokes retournés. Un mois après le début de la poke $\operatorname{War}^{20}$ elle se poursuit encore, et cette utilisation de l'application sur le long terme nous permet de mieux la comprendre.

Posture de l'espion: cette dernière posture est nommée de façon volontairement provocatrice. Le chercheur en sciences humaines et sociales qui exploite un lieu de corpus 
numérique tel que celui que nous venons de décrire n'est pas simplement un «ami Facebook» des locuteurs et un utilisateur des RSN, il doit également assumer une position de voyeur. Le terme voyeur renvoie à une exhibition passive des locuteurs et à la simple observation du voyeur, deux hypothèses non vérifiées dans notre cas. Nous l'avons $\mathrm{vu}$, les locuteurs sont actifs dans le corpus, pour une partie d'entre eux au moins. De plus, le travail de recherche demande une certaine exploitation des données observées. Raisons pour lesquelles nous préférons le terme d'espion à celui de voyeur ou à celui d'observateur qui induiraient en erreur sur la passivité du chercheur.

Il faudrait ajouter que la posture d'espion que prend le chercheur se réalise dans un contrat tacite avec les locuteurs qui sont des « espionnés » consentants. Ils acceptent le regard du chercheur en ajoutant Ma Thèse Sdl à leur liste d'amis. Toutefois, il ne s'agit pas d'exhibitionnisme puisqu'une fois qu'ils ont ajouté le profil à leur liste d'amis ils poursuivent leur existence numérique en oubliant le regard du chercheur. Le chercheurespion a également accès à des données émises avant l'intégration à la liste d'amis qui sont donc réalisées en dehors de son « regard ».

La définition de la notion de lieu de corpus nous amène à questionner chaque étape, terme et méthodologie au regard du numérique. Ces nouveaux terrains ouvrent l'accès à l'intime et permettent de rendre perméable les limites du corpus, mais ces perspectives doivent s'accompagner d'un cadre scientifique lisible et assumé.

Les technologies numériques font partie du quotidien des utilisateurs et ont généré des comportements nouveaux qui sont eux-mêmes à l'origine de nouvelles formes de discours. Pour aborder ces corpus 2.0 il faut peut-être imaginer une méthodologie 2.0 qui soit capable d'en rendre compte. C'est ce que nous avons voulu proposer dans cet article en montrant que la définition de la notion de lieu de corpus s'accompagne de réflexions sur sa représentation, sur la place des acteurs et sur la posture du chercheur.

Dans cet article nous avons tenté de proposer une définition à la notion de lieu de corpus et de considérer à la fois ce qu'elle apporte à la boite à outils linguistique et les questions qu'elle pose au linguiste. Les rapports entre le chercheur, les locuteurs et les environnements numériques sont à interroger pour chaque lieu de corpus mais quelques caractéristiques communes semblent se dessiner. C'est sur ce socle, qui va dans le sens de la mise en commun d'outils théoriques et méthodologiques pour aborder les terrains numériques, que nous avons proposé les notions de lieu de corpus, de corpus idionumérique et de représentation arborescente. Malgré tout, ces notions restent des ébauches qui posent encore beaucoup de questions, notamment leur caractère numérique et leur incapacité à représenter les formes numériquées.

\section{BIBLIOGRAPHIE}

Anderson N., Le hobo: sociologie du sans-abri suivi de L'empirisme irréductible, Nathan, Paris, 1993. 
Baude, O., « Corpus oraux les bonnes pratiques d'une communauté scientifique », dans actes du colloque Corpus en lettres et sciences sociales, des documents numériques à l'interprétation, Colloque d'Albi Langages et Signification, Presses universitaires de Toulouse, 2006, publié en 2007 (p 61-66).

Baudry, J. et Burel, F., Écologie du paysage. Concepts, méthodes et applications, Paris, TEC \& DOC, 1999, $362 \mathrm{p}$.

Bommier-Pincemin B., Diffusion ciblée automatique d'informations : conception et mise en œuvre d'une linguistique textuelle pour la caractérisation des destinataires et des documents, Thèse de Doctorat en Linguistique, Université Paris IV Sorbonne, 6 avril 1999, chapitre VII : "Caractérisation d'un texte dans un corpus : du quantitatif vers le qualitatif", § A "Définir un corpus", (p. 415-427).

Bourdeloie H., «Ce que le numérique fait aux sciences humaines et sociales », tic\&société [En ligne], vol. 7, N² 2013. Publié le 9 juin 2014, consulté le 20 juillet 2015 à l'adresse suivante : http://ticetsociete.revues.org/1500; DOI : 10.4000/ticetsociete.1500

Boutet J. et Heller, M., « Enjeux sociaux de la sociolinguistique : pour une sociolinguistique critique », Langage et société 3-4, $2007: \mathrm{n}^{\circ}$ 121-122 : 305-318.

Boyer H., « Sociolinguistique : faire corpus de toute(s) voix? ", Mots. Les langages du politique [En ligne], 69 | 2002, mis en ligne le 14 mai 2008, consulté le 27 mai 2016 à l'adresse suivante : http://mots.revues.org/10553

Bretegnier A., « Sociolinguistique alter réflexive. Du rapport au terrain à la posture du chercheur ", Cahiers de sociolinguistique $1 / 2009$ ( $\left.n^{\circ} 14\right)$, (p. 27-42). Consulté le 27/05/2016 à l'adresse suivante : www.cairn.info/revue-cahiers-de-sociolinguistique-2009-1-page-27.htm

Bretegnier, A., « Articuler recherches et interventions... pour construire une professionnalité de sociolinguiste », in ELOY \& PIEROZAK, Actes du Ve Colloque International du RFS, Intervenir : appliquer, s'impliquer?, coll. « Espaces discursifs », Paris, L’Harmattan, 2009.

Cappeau P., Gadet F., «L'exploitation sociolinguistique des grands corpus. Maître-mot et pierre philosophale», Revue française de linguistique appliquée 1/2007 (Vol. XII : p. 99-110) Consulté le 05/05/2016 à l'adresse suivante : www.cairn.info/revue-francaise-de-linguistiqueappliquee-2007-1-page-99.htm

Dacos, M. et Mounier, P., L'Édition électronique, Paris, 2010, La Découverte.

Develotte C., Mangenot, F., « L'analyse des corpus multimodaux en ligne : état des lieux et perspectives ", in Actes du congrès de l'Actualité de la recherche en éducation et en formation (AREF), Université de Genève, septembre 2010, consulté le 29/3/2016 à l'adresse suivante :

https://plone.unige.ch/aref2010/symposiums-courts/coordinateurs-en-e/professionnalite-desenseignants-se-former-dans-les-environnements-numeriques-d2019apprentissage/Feed-back \% 20correctifs.pdf

Develotte C., «L'analyse des corpus multimodaux en ligne : état des lieux et perspectives », in Actes du $3^{e}$ Congrès Mondial de Linguistique Française, 2012 (p 509-525), consulté le 3/3/2016 à l'adresse suivante : http://www.shs-conferences.org/articles/shsconf/abs/2012/01/ shsconf_cmlf12_000213/shsconf_cmlf12_000213.html

Dervin, F. et Abbas, Y. (dir.), Technologies numériques du soi et (co-)constructions identitaires, Paris, L'Harmattan, 2009.

Emerit-Bibié L., "Corpus ou lieu de corpus? ", Mémo numérique(s) [Carnet de recherche], janvier 2016, http://memo.hypotheses.org/88, consulté le 25 mars 2016. 
Emerit-Bibié L., " Le corpus idionumérique ", Mémo numérique(s) [Carnet de recherche], mars 2016, https://memo.hypotheses.org/136, consulté le 25 mars 2016.

Emerit-Bibié L., «La représentation arborescente du corpus numérique », Mémo numérique(s) [Carnet de recherche], mars 2016, http://memo.hypotheses.org/130, consulté le 25 mars 2016.

Gadet, F., « Derrière les problèmes méthodologiques du recueil des données », en ligne : Texto!, 2003, consulté à l'adresse suivante : http://www.revue-texto.net/Inedits/Gadet_Principes.html

Gadet F., Ludwig R., Mondada L., Pfänder S., Simon A-C., « Un grand corpus de français parlé : le CIEL-F. Choix épistémologiques et réalisations empiriques», Revue française de linguistique appliquée 1/2012 (Vol. XVII : p. 39-54) .Consulté le 15/05/2016 à l'adresse suivante : www.cairn.info/revue-francaise-de-linguistique-appliquee-2012-1-page-39.htm

Gumperz, J., Sociolinguistique interactionnelle. Une approche interprétative, trad. de J. Simonin, Paris, L'Harmattan, 1989.

Heller, M., Eléments d'une sociolinguistique critique, Paris, Didier, 2002.

Mayaffre D., «Corpus et Web-corpus. Réflexion sur la corporalité numérique », Cahiers de praxémique, Publications de l'Université Paul Valéry, 2010 (pp. 233-248).

Paveau M-A., « Technodiscursivités natives sur Twitter. Une écologie du discours numérique », Epistémé 9, (Revue internationale de sciences humaines et sociales appliquées), Séoul, 2013 (p 139-176).

Paveau M.-A., "Analyse discursive des réseaux sociaux numériques", Dictionnaire d'analyse du discours numérique, Technologies discursives, [Carnet de recherche], 9 mai 2013, consulté le 29/03/2016 à l'adresse suivante : http://technodiscours.hypotheses.org/?p=431

Paveau M.-A., « Environnement », Technologies discursives [Carnet de recherche], 2013. Consulté le 07 juin 2015 à l'adresse suivante : http://technodiscours.hypotheses.org/?p=311

Paveau M-A., « Ce qui s'écrit dans les environnements numériques », Itinéraires, [en ligne], 2014-1 | 2015, mis en ligne le 12 janvier 2015, consulté le 29 mars 2016. URL : http:// itineraires.revues.org/2313

Rastier,F., Pincemin, B., « Des genres à l'intertexte », Cahiers de Praxématique, Ioannis Kanellos (dir.), “ Sémantique de l'intertexte ”,n³3, 1999 (p. 83-111).

Rastier F., « Enjeux épistémologiques de la linguistique de corpus », in G. Williams (éd.), La linguistique de corpus. Rennes : PUR, 2005 (p. 31-45). [En ligne] Texto! (http://www.revuetexto.net/Inedits /Rastier/ Rastier_Enjeux.html

Robillard D. (de), « La linguistique autrement : altérité, expérienciation, réflexivité, constructivisme : en attendant que le Titanic ne coule pas ", CAS, 1, 50 ans après le Cours de Saussure : la linguistique en question, Université de Picardie, 2007 (p. 81-228).

Sinclair J., Corpus, Concordance, Collocation, Oxford, Oxford University Press Esp, 1991.

Sinclair, J., Preliminary recommendations on Coprus Typology. Technical report, EAGLES, (Expert Advisory Group on Language Engineering Standards), 1996, consulté le 18 mars 2016 à l'adresse suivante : http://www.ilc.cnr.it/EAGLES96/pub/eagles/corpora/corpustyp.ps.gz

Theviot A., « Devenir « ami » avec 4500 enquêtés. Les enjeux éthiques de l'analyse d'interfaces semi-privées ", tic\&société [En ligne], vol. 7, N 2, 2013. Publié le 01 juin 2014, consulté le 20 juillet 2015 à l'adresse suivante $: \underline{\text { http://ticetsociete.revues.org/1608; DOI : 10.4000/ticetsociete.1608 }}$ Vrancken D, Kuty O. (éds.), La sociologie de l'intervention. Enjeux et perspectives, Bruxelles, De Boeck Université, 2001. 


\section{NOTES}

1. Par exemple à partir d'un téléphone, d'une tablette ou d'un ordinateur.

2. Description du Discours Numérique : Étude des bouleversements linguistiques du web 2.0 au travers de l'exemple des souhaits d'anniversaire sur Facebook. Thèse soutenue le 27 novembre 2015 à l'université Bordeaux-Montaigne.

3. Le terme affordance apparait en psychologie, il est utilisé par Gibson (1979) pour désigner « la relation de réciprocité entre des acteurs et un environnement, une articulation entre ce qui est permis par l'outil et la variété d'emplois que les utilisateurs vont en faire.» (Develotte 2012 : 514). Il est parfois remplacé par " potentialités » dans la littérature mais on y perd la notion de réciprocité entre les acteurs et leur environnement qui est centrale ici. Nous ferons donc la différence dans cet article entre les potentialités qui concerne l'aspect technologique et les affordances qui ajoutent la dimension relationnelle.

4. Il s'agit d'une page d'accueil sur laquelle apparaissent, dans l'ordre antéchronologique, les messages publiés par les « amis » de l'utilisateur.

5. Cette précision est nécessaire puisque les constituants de cet environnement sont en évolution permanente (ajout ou retrait de contact à la liste d'amis notamment).

6. Ou « mises en avant " pour reprendre le métadiscours de Facebook.

7. Affichage des données, mise en avant de publicités, etc.

8. Accès au site par une application ou par le web.

9. Réseaux sociaux numériques.

10. Puisque c'est avant tout pour représenter Ma thèse Sdl dans le cadre d'une recherche doctorale que cette modélisation a été réalisé.

11. Les utilisateurs de Facebook peuvent consulter les publications des autres membres directement sur leurs journaux ou à partir de certaines applications du site qui les regroupent en fonction d'algorithmes comme par exemple le fil d'actualités.

12. Avec les moyens actuels, on peut tout à fait imaginer que les choses évoluent et qu'il soit un jour possible d'aller analyser directement les données dans leurs environnements numériques d'apparition.

13. Au moment de l'extraction.

14. La présence de cette adresse URL n'est pas obligatoire. Elle est générée automatiquement par le site lorsqu'un contenu est partagé à l'aide de la fonction copier-coller mais elle peut être effacée sans que le contenu disparaisse.

15. Traitement automatique des langues

16. http://www.iramuteq.org/

17. http://textometrie.ens-lyon.fr/

18. Notamment pour faciliter les repérages dans le corpus grâce aux fonctions de base du logiciel qui permettent de créer un lexique et de repérer les cooccurrences.

19. Ils ont la possibilité de quitter le lieu de corpus à tout moment ce qui a un impact sur toutes les données.

20. Poke War est le nom donné à la pratique qui consiste à envoyer et recevoir des pokes, par les utilisateurs de Facebook. L'objectif de la poke War est d'être le dernier utilisateur à envoyer un poke. 


\section{RÉSUMÉS}

Cet article propose de considérer la notion de lieu de corpus comme une alternative numérique à celle de corpus. Le lieu de corpus est un espace numérique délimité dont les données possèdent trois caractéristiques incompatibles avec la notion de corpus: l'instabilité, la mixité et l'incomplétude. La définition de cette notion s'appuiera sur la description du compte Facebook «Ma Thèse Sdl », lieu de corpus constitué dans l'objectif d'observer le discours produit sur les réseaux sociaux numériques. Elle sera complétée par la proposition d'une méthodologie de représentation du lieu de corpus: la représentation arborescente. Enfin, les nouvelles perspectives d'interactivité ouvertes par cette notion permettront d'interroger la place des acteurs le constituant et la posture du chercheur.

This article proposes to consider corpus-place concept as a digital alternative to the corpus notion. Corpus-place is a delimited digital data space with three characteristics incompatible with the notion of corpus : instability, diversity and incompleteness. Definition of this concept will be based on the description of Facebook account "Ma Thèse Sdl " corpus-place build with the objective of observing discourse produced on digital social networks. It will be completed by the proposal of a corpus-place representation methodology : tree representation. Finally, the new interactive opportunities offered by this concept will question place of actors and posture of researcher.

\section{INDEX}

Mots-clés : Lieu de corpus, Analyse du discours numérique, Interactions par écran, représentation arborescente, corpus idionumérique, corpus multimodal, posture du chercheur. Keywords : Corpus-place, digital speech analysis, screen interactions, tree representation, idionumérique corpus, multimodal corpus, researcher posture.

\section{AUTEUR}

\section{LAETITIA EMERIT}

Université Bordeaux Montaigne

CLLE ERSSàB

<emerit.laetitia@gmail.com> 